\title{
Personal Control, Social Problem-Solving and Social Adjustment in Obsessive-Compulsive Disorder
}

Melanie Aubut and Claude Belanger*

Department of Psychology, University of Quebec in Montreal, Montreal, Canada

\begin{abstract}
Different studies conducted among obsessive-compulsive disorder (OCD) patients explain the relevance of investigating social adjustment impairments due to its pervasiveness in a patient's occupational, recreational and/or interpersonal functioning. Upon careful examination of the social adjustment contributing factors, both personal control and social problem-solving were identified as variables that influence the adjustment capacities in different clinical groups. Nevertheless, few studies have examined these variables among OCD patients. This study aimed to examine the relationship between the aforementioned factors in an OCD sample group $(n=128)$ and a control group ( $n=235)$. The results showed that OCD patients exhibited more of an external locus of control than did the control group. OCD patients also demonstrated a significant impairment in overall social problem-solving skills compared to their control counterparts. Moreover, a negative problem orientation itself has been found to mediate the relationship between the locus of control and social adjustment. Overall, these results convey the importance of social problem-solving in understanding altered social functioning among OCD patients.
\end{abstract}

Keywords: Personal control; Social problem-solving; Social adjustment; Obsessive-compulsive disorder

\section{Introduction}

The clinical picture of people with obsessive-compulsive disorder (OCD) is characterized by recurrent obsessions and/or compulsions [1] and functional alterations that are observable in all areas of life [2]. Epidemiological data from several Canadian and American studies further support the association between social adjustment difficulties and obsessive-compulsive symptoms [3-6]. Indeed, people with OCD present a more altered but otherwise similar level of functioning than do patients with other psychopathologies [7-11]. Our on-going research on these individuals' social adjustment, i.e., their ability to adapt to their social roles and to obtain satisfaction from them [12], has also confirmed the importance of observed adjustment difficulties and their generalization to all areas of functioning. Factors that explain these social adjustment difficulties observed in individuals with OCD have yet to be specified. However, efforts to improve our understanding of these difficulties have identified two variables associated with individuals' social adjustment abilities and the presence of clinical disorders such as OCD: personal control (PC) and social problem-solving (SPS).

\section{Personal Control}

Locus of control [13] or personal control [14] refers to the "location" of control is defined by two underlying dimensions: The internal (ILC) and the external (ELC) locus of control. People with a predominant internal locus of control tend to believe they can influence the course of events in which they are involved, whereas those with an external locus of control instead believe that events are influenced by external factors and that they have little or no influence on these events [13]. Some authors consider it a problem to only focus on the location of control since it is not sufficient to believe that actions are responsible for results; it is also essential to consider an individual's perceptions about his or her ability to control his or her actions [15]. Thus, Bandura juxtaposed the concept of locus of control with efficacy, that is, one's belief in one's ability to effectively perform a certain behavior in pursuit of a desired goal. Researchers in the field of locus of control also mention the concepts of sense of control and desire for control. Whereas sense of control refers to an individual's belief in his or her power to perform an action and produce a result, desire for control consists of the individual's motivation to have control [16]. Njus and Brockway argue that the perception of social skills explains much more variance in adjustment than what is explained by locus of control [17]. Orientations towards problems and SPS styles could, by their resemblance to the notions of efficacy and competence, play a role in the relationship between locus of control and social adjustment.

The notion of locus of control can explain the intra- and inter-group differences in the ability to adapt to different contexts. It is generally supported that a low locus of control is more prevalent in different clinical groups [18] and is generally associated with lower emotional or social adjustment [19]. An internal locus of control is negatively associated with psychological distress [20] and appears beneficial to psychological adjustment [21]. It is also proposed that extreme and biased belief in PC, both external and internal, is associated with adjustment problems because extreme beliefs are usually unrealistic $[8,22]$. Furthermore, an external locus of control is positively associated with social and emotional adjustment [23]. This challenges the widespread notion that it is always beneficial to maintain an internal locus of control and that maintaining an external locus of control denotes maladjustment.

Although the role of PC has been widely studied in other disorders, to our knowledge, few studies have examined its role in relation to OCD. However, the notion of control is important for those people. Indeed, the fear of losing control of their thoughts and/or actions is often present and the need to control them through compulsions is important [24]. The few articles that have been reviewed, however, show that some trends tend to emerge in people with OCD. A more important external locus of control was found in such individuals when compared to controls $[25,26]$ but was found to be equivalent to other clinical groups [27].

A few studies have suggested that in people with OCD, PC could

*Corresponding author: Claude Bélanger, Department of Psychology, University of Quebec in Montreal, C.P. 8888 Centre-ville, Montreal (Quebec) H3C 3P8, Tel: (514) 987-3000 Ext. 1777; E-mail: belanger.claude@uqam.ca

Received March 21, 2017; Accepted April 27, 2017; Published May 05, 2017

Citation: Aubut M, Belanger C (2017) Personal Control, Social Problem-Solving and Social Adjustment in Obsessive-Compulsive Disorder. J Psychol Abnorm 6: 156. doi: 10.4172/2471-9900.1000156

Copyright: ( 2017 Aubut M, et al. This is an open-access article distributed under the terms of the Creative Commons Attribution License, which permits unrestricted use, distribution, and reproduction in any medium, provided the original author and source are credited. 
have an impact on OCD symptoms via its interaction with other variables. It was first argued that a more severe obsessive-compulsive symptomatology is associated with a low sense of control towards adverse life events [28]. Individuals with a strong desire for control, combined with a lower assessment of their level of control, also tend to experience more severe obsessive-compulsive symptoms [29]. Altin and Karanci also suggest that there is an interaction between the effect of responsibility attitudes and locus of control on symptomatology, with an exaggerated sense of responsibility and an external locus of control producing the strongest symptoms [30].

The examination of these results suggests that some factors associated with PC may affect the understanding of OCD and associated social adjustment difficulties. Although PC appears to be an important construct in the understanding of a person's interactions with the world, other variables may also account for adjustment, such as SPS. Thus, one might think that the association between PC and social adjustment is influenced by variables that address the ability to "act".

\section{Social Problem-Solving}

Social problem-solving (SPS) is the process by which a person attempts to identify efficient and adaptive solutions to everyday problems. D'Zurilla et al. identify two adaptive dimensions of SPS (i.e., positive orientation and rational problem-solving) and three dysfunctional dimensions (i.e., negative orientation, impulsive/ careless style and avoidant style) towards problems. Positive problem orientation was described as a SPS constructive cognitive pattern that involves different general dispositions: 1) viewing problems as challenges rather than threats, 2) believing that problems are solvable, 3) believing in one's ability to solve problems successfully, 4) believing that successful problem-solving requires time, effort and persistence and 5) committing to solving problems promptly rather than avoiding them. In contrast, negative problem orientation is a dysfunctional cognitive and affective pattern that involves general trends such as the disposition to consider a problem as a significant threat to one's well-being, doubting one's ability to solve problems successfully, and becoming frustrated when confronted with a problem [31].

Rational problem-solving is defined by the rational, deliberate, systematic and clever application of effective and adaptive principles and techniques. This problem-solving style is divided into four subscales: 1) problem definition and formulation, 2) decision making, 3) generation of alternative solutions and 4) solution implementation and verification. In contrast, the impulsive/careless style of problemsolving is characterized by active attempts to apply limited, careless, precipitated or incomplete strategies or techniques. Finally, the avoidant style of problem-solving is characterized by procrastination, passivity or dependence, which leads people to avoid problems rather than confront them, delay the resolution as much as possible, wait for problems to resolve by themselves, and attempt to transfer the responsibility for the problem resolution to others [31].

Researchers have consistently found a relationship between problem-solving deficits and psychological distress, especially for patients presenting depressive and anxious symptoms [32]. Individuals with effective problem-solving strategies report less depression, anxiety, problems and distress when dealing with them [33]. Similarly, problem-solving is positively associated with psychological well-being $[34,35]$ Studies that are interested in the specific influence of SPS on adjustment [36] suggest that negative problem orientation is associated with concern $[37,38]$ and that SPS orientations and styles are correlated to anxiety and depression [39]. Furthermore, perfectionism and SPS are related to depression and suicidal ideation, where SPS protects the individual from the negative impact generally associated with perfectionism [40].

In the same vein, problem-solving was identified as a mediator in the relationship between everyday problems and anxiety [39] and as a moderator of the adverse effects resulting from stressful life events on depression [41] and anxiety [42,43]. For SPS, it was shown that negative and positive problem orientation as well as impulsive/careless and avoidant styles are all mediators or moderators depending on the gender of the participants and of the dimensions studied in the relationship between stress and internalized and externalized symptoms [44]. Other researchers have argued that the constructive dimensions of SPS are positively related to psychological well-being, that the three dysfunctional scales of SPS are negatively related to psychological well-being, and that SPS acts as a partial mediator in the relationship between stress and well-being [45].

These results support the relationship between SPS and psychological difficulties. In light of the preceding results, it is possible that the dimensions of SPS may also have an impact on obsessive-compulsive symptoms and adjustment in the affected people. However, few studies have explored these associations in individuals presenting with OCD. The results indicate that these individuals show significantly more negative patterns of SPS (i.e., negative problem orientation, impulsivity/ careless style and avoidance style) and fewer positive patterns (i.e., positive problem orientation and rational problem solving) compared to controls [46]. Furthermore, negative problem orientation has also been associated with symptoms of generalized anxiety and OCD in a community-based sample of university students [47]. However, the manner in which these variables relate in individuals diagnosed with OCD or in those experiencing remission of this disorder has yet to be explained.

\section{Personal Control and Social Problem-Solving}

Personal control (PC), which is comparable to locus of control (LC) and social problem-solving (SPS), are two variables that, in light of the scientific literature, appear to affect an individual's coping skills. Moreover, studies support the presence of associations between the locus of control and problem-solving orientation [33] as well as the interactions between these concepts and several psychological or social adjustment variables [48]. Indeed, an external locus of control is associated with perceived inefficiency of SPS [33] and an internal locus of control with better problem-solving skills [49]. Perceived control was positively associated with problem-oriented adaptation strategies [50] and individuals considering their problem-solving skills to be effective tend to attribute the causes of their problems to internal and controllable factors [51]. All of these results support the idea that PC affects one's problem-solving style and orientation, which in turn has a significant impact on an individual's adjustment abilities. It would therefore be interesting to see whether this pattern can be observed in people suffering from OCD.

\section{Objectives and Hypotheses}

The aim of this study is to clarify the relationships between personal control (PC), the social problem-solving (SPS) style and orientation, and social adjustment in people suffering from OCD. This will allow the determination of the extent to which PC and SPS are associated with the development and maintenance of social adjustment difficulties in people with OCD. Thus, the first objective of the study is to compare PC and dimensions of SPS between an OCD group and a control group. It is hypothesized that the OCD group will demonstrate more of an external locus of control and more negative patterns of SPS than the control 
group. The second objective of the study is to analyze the relationships between PC and global social adjustment. For both groups, it is expected that an external locus of control will be associated with more important adjustment difficulties. This study will also examine the links between SPS and social adjustment. It is hypothesized that more negative patterns of SPS will be associated with more severe adjustment difficulties. The study will also facilitate the analysis of the relationships between PC and the SPS style and orientation. It is presumed that for both groups, an external locus of control will correlate with dysfunctional SPS styles and orientations. Finally, the study will examine the mediational role of SPS in the relationship between PC and global adjustment; it is expected that the effect of the independent variable will be eliminated or significantly reduced after controlling for the mediator.

\section{Method}

\section{Participants}

First, a group of 128 participants ( 92 women, 36 men) who received a primary diagnosis of OCD and were between 18 and 65 years of age were recruited through wide publicity of the project in the local media. They were also recruited from hospital mental health clinics, specialized or psychological clinics, various academic settings, and through several community organizations that assist people with OCD. The presence of a comorbid psychiatric disorder and psychotropic medication is not considered exclusion criteria for this study. A control group of 235 people between 18 and 65 years old were recruited from classes at the University of Quebec in Montreal.

\section{Instruments}

\section{Sociodemographic measures}

The General Information Questionnaire was developed by our team to collect sociodemographic information such as gender, age, occupation, income, education and marital status. Due to organizational constraints and restrictions associated with the recruitment site, only information about gender and age could be asked and was available for the control group.

\section{Obsessive-compulsive disorder diagnosis}

The presence and severity (i.e., mild, moderate and severe) of OCD was measured with the Anxiety Disorders Module for clinical interviews of the SCID-I/P [52]. This interview also provided information on the presence of other anxiety disorders in the past month and psychotropic medications. This interview was not administered to the control group.

\section{Social adjustment measure}

Social adjustment was evaluated with the validated French version [53] of the Social Adjustment Scale-Self-Report [54]. The reliability and validity for the instrument were supported in several scientific articles. The internal consistency measures, test-retest coefficients and sensitivity to treatment effects are all considered good [55]. In addition to assessing global social adjustment, the measure evaluates adjustment in six areas of life: 1) work (worker, homemaker or student), 2) social and leisure activities, 3 ) relationships with the extended family, 4) role as a marital partner, 5) parental role, and 6) role within the family unit. For this measure, low scores are considered desirable and represent better adjustment.

\section{Social problem-solving measure}

The validated French version [38] of the Social Problem-Solving Inventory-Revised was used. The evaluation of the psychometric properties of the original instrument supports strong internal consistency, temporal stability as well as concurrent, predictive, convergent and discriminant validity [31]. This measure gives a total score and scores for five scales: 1) positive and 2) negative problem orientation 3) rational problem-solving, 4) impulsive/careless style towards problems and 5) avoidant style towards problems. The rational problem-solving scale comprises four subscales: 1) problem definition and formulation, 2) decision making, 3) generation of alternative solutions and 4) solution implementation and verification. The scores are reversed for the negative problem orientation, avoidant style and impulsive/careless style scales, with lower scores representing greater difficulty for these dimensions.

\section{Personal control measure}

The French version of the Belief in Personal Control Scale-Revised Short Form was also administered to both groups. The original English scale showed good psychometric properties. Our team translated English version into French and performed reliability analyses. The Cronbach's alphas are 0.848 for the general external control scale and 0.818 for the exaggerated internal control scale, which suggests good internal consistency for both scales. This measure evaluates three dimensions of PC, but only the first two dimensions were administered: general external control and exaggerated internal control. General external control measures the extent to which an individual believes life events result from internal or external factors. Higher scores on this scale represent a more internal locus of control. Exaggerated internal control is a measure of extreme and unrealistic beliefs in one's PC and represents a dimension of control that is characterized by, at its upper limit, excessive optimism or extreme confidence into one's own power to control events [14].

\section{Procedure}

The ethics committees of all the institutions in which the recruitment was held approved the research. The OCD group participants were interviewed with a structured diagnosis instrument, the SCID-I/P [52], to assess the presence and severity of OCD. The interviews were conducted by research assistants, all of whom were doctoral students in psychology who were trained in the assessment of anxiety disorders and who received advanced training in the administration of the SCID-I/P. All participants were also asked to complete the research questionnaires.

\section{Results}

\section{Sociodemographic and clinical data}

For the group of OCD-diagnosed participants, the exploration of the data shows that the symptoms are mild (17\%), moderate $(45 \%)$ or severe $(38 \%)$. Additionally, $38.3 \%$ presented with a secondary anxiety disorder in the previous month. However, because no association was found between these secondary anxiety disorders and adaptation, it was not considered a confounding variable. The average age of the participants in the OCD group, which was mainly composed of women (72\%), is 39.77 years (SD 12.48); $52 \%$ are living in a common-law relationship or are married, $11 \%$ are separated or divorced, $36 \%$ are single and $1 \%$ widowed. In addition, $4 \%$ have completed only primary education, $29 \%$ secondary education and $30.5 \%$ collegiate education, whereas $30.5 \%$ have an undergraduate degree and $6 \%$ have a graduate degree. For occupation, $45 \%$ report having a full-time job, $23 \%$ have a part-time job, $32 \%$ report having no job and $14 \%$ report being parttime or full-time students. The control group was mainly composed of women $(76 \%)$, which is similar to the OCD sample. The group 
participants are between 18 and 59 years old and are significantly younger $(\mathrm{M}=25.1, S D=7.8)$ than the current OCD group $(\mathrm{M}=39.77, S D=12.48$, $t(298)=12.49, p<0.05)$. Nevertheless, the univariate analyses show that neither gender nor age are significantly associated with global social adjustment (both $p>0.05, n s$ ); accordingly, these sociodemographic variables were not considered potential confounding variables.

\section{Intergroup differences in personal control and social problem-solving}

The variables in the study were first analyzed to verify the correspondence between the variables' distribution and the assumptions of the completed analyses. The analysis of $\mathrm{Z}$-scores for each variable revealed ten univariate outliers; these values were deleted, reducing the total sample from 363 (128 OCD and $235 \mathrm{CTRL}$ ) to 353 participants (122 OCD and 231 CTRL).

A multivariate analysis of variance (MANOVA) was performed with seven dependent variables: 1) general external control 2) exaggerated internal control 3) negative problem orientation 4) positive problem orientation 5) avoidant style 6) impulsive/careless style and 7) problem definition and formulation. The independent variable is the group (OCD and CTRL). All the variables that were entered in the multivariate analysis were significantly correlated to at least one other variable at $r>0.3$, with the coefficients ranging from $r=0.06$ to 0.55. A multivariate outlier was identified in the control group and was deleted with $\chi^{2}(7)=24.322, \mathrm{p}<0.001$. For the MANOVA analysis, the total sample was thus reduced to 278 participants ( 70 OCD and 208
CTRL) with the removal of outliers and missing values for the variables included. The evaluation of the assumptions for these analyses shows satisfactory results.

With the use of Wilks' Lambda criterion, a main effect of the group on the dependent variables is observed $\mathrm{F}(7,270)=7.56, \mathrm{p}<0.001$. Subsequent between subject tests indicate a significant effect of the group on all dependent variables (all $\mathrm{p}<0.01$ ), except for the impulsive/ careless style and avoidant style variables (both $\mathrm{p}>0.05$, ns). Therefore, people in the OCD group present lower score for all variables, but the groups do not differ with regard to the impulsive/careless and avoidant styles. The results from the between-subject tests and descriptive data are presented in (Tables 1 and 2).

Post hoc analyses were performed using GPower [56] to assess the possibility that the non-significant results may be due to a lack of statistical power. Calculations for ANOVA with $\alpha=0.05, n=278$ and two groups showed that the chances of detecting moderate and large effects were adequate (0.99) but at only $38 \%$ and were unsatisfactory for detecting small effect sizes. Consequently, caution should be used with non-significant results.

T-tests were performed with the variables excluded from the MANOVA to avoid multicollinearity problems: 1) the SPS total score 2) rational problem-solving 3) decision making 4) generation of alternative solutions and 5) solution implementation and verification. The independent variable is the group (OCD and CTRL). The results from the T-tests and descriptive data are presented in Table 3. First, the

\begin{tabular}{|c|c|c|c|c|}
\hline \multirow{2}{*}{ Measures } & \multicolumn{2}{|c|}{ OCD } & \multicolumn{2}{|c|}{ CTRL } \\
\hline & M & SD & M & SD \\
\hline General external control $^{a}$ & $65.52^{\mathrm{a}}$ & 9.06 & $71.31^{\mathrm{a}}$ & 7.56 \\
\hline Exaggerated internal control ${ }^{b}$ & $54.37^{\mathrm{b}}$ & 6.86 & $57.86^{b}$ & 7.09 \\
\hline Negative problem orientation $^{c}$ & $19.50^{c}$ & 9.43 & $27.52^{\mathrm{c}}$ & 7.08 \\
\hline Positive problem orientation $^{d}$ & 10.10 & 3.99 & 12.17 & 3.72 \\
\hline Problem definition and formulation & 10.69 & 4.11 & 11.96 & 3.59 \\
\hline Impulsive/careless style ${ }^{c}$ & $31.07^{c}$ & 6.83 & $31.89^{c}$ & 6.03 \\
\hline Avoidant style ${ }^{c}$ & $19.40^{c}$ & 5.87 & $21.74^{c}$ & 4.31 \\
\hline
\end{tabular}

Note: Means and standard deviations on non-transformed variables. OCD: Obsessive-Compulsive Disorder; CTRL: Control. a-Higher scores represent more internal control on an external/internal continuum. b-Higher scores represent more unrealistic beliefs concerning exaggerated internal control. c-Lower scores represent more important difficulties in this dimension

Table 1: Means and standard deviations for personal control and social problem-solving scales included in the multivariate analysis of variance (MANOVA).

\begin{tabular}{|c|c|c|c|c|c|c|c|c|}
\hline \multirow{2}{*}{ Variables } & \multirow{2}{*}{ MANOVA F(7,270) } & \multicolumn{5}{|c|}{ ANOVA F (1,276) } \\
\cline { 3 - 7 } & & GEC & EIC & NPO & PPO & PDF \\
\hline Group & $7.56^{* *}$ & $31.67^{* *}$ & $14.53^{* *}$ & $29.92^{* *}$ & $12.73^{* *}$ & $7.51^{*}$ & 1.06 \\
\hline
\end{tabular}

Notes: F ratios are Wilks' F estimates. ANOVA: Analysis of Variance; MANOVA: Multivariate Analysis of Variance; GEC: General External Control; EIC: Exaggerated Internal Control; NPO: Negative Problem Orientation; PPO: Positive Problem Orientation; PDF: Problem Definition and Formulation; ICS: Impulsive/Careless Style; AS: Avoidant Style

${ }^{*} \mathrm{p}<0.01$

${ }^{* *} \mathrm{p}<0.001$

Table 2: Univariate and multivariate analyses of variance for personal control and social problem-solving scales.

\begin{tabular}{|c|c|c|c|c|c|c|c|c|}
\hline \multirow{2}{*}{ Variables } & \multicolumn{2}{|c|}{ OCD } & \multicolumn{2}{|c|}{ CTRL } & \multirow{2}{*}{ dF } & \multirow{2}{*}{$\mathbf{t}$} & \multirow{2}{*}{$\mathbf{p}$} & \multirow{2}{*}{ Cohen's d } \\
\hline & M & SD & M & SD & & & & \\
\hline Problem solving global score & 11.93 & 2.71 & 13.75 & 2.36 & 301 & -5.64 & 0.000 & -0.65 \\
\hline Rational problem solving & 39.98 & 15.35 & 45.90 & 12.30 & 144.30 & -3.30 & 0.001 & -0.55 \\
\hline Decision making & 9.98 & 4.58 & 11.94 & 3.60 & 150.41 & -3.74 & 0.000 & -0.61 \\
\hline Generation of alternative solutions & 9.72 & 4.37 & 11.36 & 3.32 & 146.36 & -3.30 & 0.001 & -0.55 \\
\hline $\begin{array}{c}\text { Solution implementation and } \\
\text { verification }\end{array}$ & 9.39 & 4.58 & 10.73 & 3.77 & 155.38 & -2.54 & 0.012 & -0.41 \\
\hline
\end{tabular}

Notes: Means and standard deviations on non-transformed variables/analyses on normalized variables. OCD: Obsessive-Compulsive Disorder; CTRL: Control

Table 3: Intergroup differences between OCD and control groups, for problem-solving global score, rational problem-solving, decision making, generation of alternative solutions and solution implementation and verification. 
results show that the participants from the OCD group have significantly lower SPS total scores than participants in the control group, $\mathrm{t}(301)=-$ 5.64, $\mathrm{p}<0.001, \mathrm{r}=0.31$. This is also the case for the SPS subscales; the participants from the OCD group have significantly lower scores than the controls for rational problem-solving, $\mathrm{t}(144.30)=-3.30, \mathrm{p}=0.001$, $\mathrm{r}=0.26$, decision making, $\mathrm{t}(150.41)=-3.74, \mathrm{p}<0.001, \mathrm{r}=0.29$, generation of alternative solutions, $\mathrm{t}(146.36)=-3.30, \mathrm{p}=0.001, \mathrm{r}=0.26$ and solution implementation and verification, $\mathrm{t}(155.38)=-2.54, \mathrm{p}=0.012, \mathrm{r}=0.20$ (Table 3).

\section{Mediation Analyses}

Before carrying out the analyses, the included variables were analyzed to verify whether the assumptions for the mediation analyses were met, and they were. For the OCD group, the analysis of the Z-scores for each variable allowed the detection of a univariate outlier that was deleted. No multivariate outlier was identified in the regression analyses performed with $\chi^{2}(2)=13.82, p<0.001$. The removal of the outlier and

\begin{tabular}{|c|c|c|}
\hline Measures & $\begin{array}{c}\text { OCD } \\
\text { Social adjustment }^{a}\end{array}$ & $\begin{array}{c}\text { CTRL } \\
\text { Social adjustment }{ }^{\mathrm{a}}\end{array}$ \\
\hline General external control $^{b}$ & $-0.343^{* * *}$ & $-0.247^{* * *}$ \\
\hline Exaggerated internal control ${ }^{c}$ & $-0.273^{* *}$ & $-0.191^{* *}$ \\
\hline Problem-solving global score & $-0.283^{* *}$ & $-0.343^{* * *}$ \\
\hline Negative problem orientation ${ }^{d}$ & $-0.418^{* * *}$ & $-0.381^{* * *}$ \\
\hline Positive problem orientation & $-0.203^{*}$ & $-0.316^{\star \star *}$ \\
\hline Rational problem-solving & -0.084 & -0.132 \\
\hline Problem definition and & -0.138 & $-0.153^{*}$ \\
\hline \multicolumn{3}{|l|}{ formulation } \\
\hline Decision making & -0.059 & -0.068 \\
\hline Generation of alternative & -0.014 & -0.079 \\
\hline \multicolumn{3}{|l|}{ solutions } \\
\hline Solution implementation and & -0.053 & $-0.163^{*}$ \\
\hline verification Impulsive/careless style ${ }^{d}$ & $-0.244^{*}$ & $-0.148^{*}$ \\
\hline Avoidant style ${ }^{d}$ & -0.186 & $-0.223^{\star * *}$ \\
\hline
\end{tabular}

Note: OCD: Obsessive-Compulsive Disorder; CTRL: Control. a-Lower scores represent better social adjustment. b-Higher scores represent more internal control on an external/internal continuum. c-Higher scores represent more unrealistic beliefs concerning exaggerated internal control. dLower scores represent more important difficulties in this dimension

${ }^{*} \mathrm{p}<0.05{ }^{* *} \mathrm{p}<0.01{ }^{* * *} \mathrm{p}<0.001$

Table 4: Correlations for personal control, social problem-solving and global social adjustment depending on the group. the presence of missing values in the included variables changed the sample sizes from 74 to 92 depending on the regression performed. For the control group, the analysis of $\mathrm{Z}$-scores for each variable allowed for the detection of seven univariate outliers that were deleted. No multivariate outlier was identified in the regression analyses that used $\chi^{2}(2)=13.82, p<0.001$. The removal of the outliers and the presence of missing values in the included variables changed the sample sizes from 206 to 212 depending on the regression performed. Correlational analyses were then executed, with the previously processed variables and for the two separate groups, to test the relationships between global social adjustment and the variables of PC and SPS. The sample sizes varied according to the correlational analyses performed, from 85 to 105 for the OCD group and from 211 to 227 for the control group. The bivariate correlations are presented in Table 4 . These analyses show that both variables for PC are significantly correlated to global social adjustment in both groups (all $p<0.01$ ). Similarly, some SPS variables show moderate effect sizes when correlated to global social adjustment; for the OCD group, negative problem orientation $(\mathrm{r}[99]=-0.42)$ and the SPS total score $(\mathrm{r}[88]=-0.28)$, at $p<0.01$; for the control group, negative problem orientation $(\mathrm{r}[218]=-0.38)$, the SPS total score $(\mathrm{r}$ [213]=$34)$ and positive problem orientation $(\mathrm{r}[218]=-32)$, at $p<0.001$. These variables were included in the subsequent mediation analyses since, in addition to presenting significant correlation coefficient effect sizes when correlated to social adjustment, they were also identified in the scientific literature as mediators in different psychological adjustment models [45,39] (Table 4).

Correlational analyses were then performed to test the relationships between PC and the selected potential mediators. Since this study aimed to examine the relationships between PC and SPS styles and orientations, correlation analyses were extended to all SPS dimensions. The sample sizes varied according to the correlational analyses performed, from 82 to 95 for the OCD group and from 206 to 217 for the control group. The results are presented in Table 5. According to the results, general external control and exaggerated internal control are, in both groups, strongly correlated to all potential mediators, which are global SPS as well as positive and negative problem orientation (all $\mathrm{p}<0.05)$. Distinctions also emerge from the SPS subscales depending on groups and the type of control (Table 5). Indeed, the PC is generally more associated with the SPS subscales in the control group, with general external control significantly associated with all subscales (all $\mathrm{p}<0.05)$ and exaggerated internal control significantly correlated to all

\begin{tabular}{|c|c|c|c|c|}
\hline Measures & OCD General external & OCD Exaggerated & CTRL General external & CTRL Exaggerated \\
\hline & Controla & Internal control ${ }^{b}$ & Controla $^{a}$ & Internal control ${ }^{b}$ \\
\hline Problem-solving global score & $0.518^{* * *}$ & $0.521^{\star * *}$ & $0.452^{* * *}$ & $0.518^{* * *}$ \\
\hline Negative problem orientation $^{c}$ & $0.508^{\star * *}$ & $0.386^{* * *}$ & $0.419^{* * *}$ & $0.482^{* * *}$ \\
\hline Positive problem orientation & $0.256^{*}$ & $0.554^{* * *}$ & $0.317^{* * *}$ & $0.549^{* * *}$ \\
\hline Rational problem-solving & $0.212^{*}$ & $0.244^{*}$ & $0.171^{*}$ & $0.392^{* * *}$ \\
\hline Problem definition and & $0.341^{* * *}$ & $0.294^{* *}$ & $0.147^{*}$ & $0.247^{* \star *}$ \\
\hline \multicolumn{5}{|l|}{ formulation } \\
\hline Decision making & 0.070 & 0.132 & $0.133^{*}$ & 0.073 \\
\hline Generation of alternative & 0.058 & 0.187 & $0.151^{*}$ & $0.362^{* * *}$ \\
\hline \multicolumn{5}{|l|}{ solutions } \\
\hline Solution implementation and & 0.130 & $0.257^{*}$ & $0.158^{*}$ & $0.190^{* *}$ \\
\hline verification Impulsive/careless stylec & $0.422^{* * *}$ & 0.059 & $0.314^{* * *}$ & -0.019 \\
\hline Avoidant stylec & $0.397^{* * *}$ & $0.411^{* * *}$ & $0.373^{* * *}$ & $0.336^{* * *}$ \\
\hline
\end{tabular}

Notes: OCD: Obsessive-Compulsive Disorder; CTRL: Control. a-Higher scores represent more internal control on an external/internal continuum. b-Higher scores represent more unrealistic beliefs concerning exaggerated internal control. c-Lower scores represent more important difficulties in this dimension

${ }^{*} p<0.05{ }^{* *} p<0.01{ }^{* * *} p<0.001$ 
subscales (all $\mathrm{p}<0.01$ ) except for decision making and the impulsive/ careless style (both $\mathrm{p}>0.05$, ns). For the OCD group, all scales are associated with PC (all $\mathrm{p}<0.05)$, except the decision making and the generation of alternative solution scales (both $\mathrm{p}>0.05$, ns). Likewise, the solution implementation and verification scale is not associated with general external control, and the impulsive/careless style is not related to exaggerated internal control ( $\mathrm{p}>0.05$, ns) (Table 5).

To assess mediation, these three conditions must be met [57]: (1) the independent variable must be related to the mediator in the first equation; (2) the independent variable must affect the dependent variable in the second equation; and (3) the mediator must affect the independent variable in the third equation. The preceding correlational analyses ensure that these three conditions have been met for every mediation analysis. After these conditions have been met, the effect of the independent variable must be eliminated or significantly reduced after controlling for the mediator. To verify that the effect of PC, through the general external control scale, on social adjustment is eliminated or reduced after controlling for SPS dimensions, hierarchical regression analyses were performed. The variables included in these analyses are negative problem orientation and SPS total score for both groups. Positive orientation was also included in the CTRL group.

None of the control variables examined in the OCD group were included in the equations since the univariate analyses indicated that none (i.e., presence of an anxiety disorder during the previous month, gender, age and OCD severity) is significantly associated with global social adjustment (all $p>0.05, n s$ ). Taking psychotropic medication, which is present in $68.8 \%$ of this group's participants, is weakly but significantly associated with social adjustment ( $\mathrm{r}$ [101]=$0.211, p=0.03$ ). However, the exploratory analyses conducted show that every mediation model remains intact when including this variable, which was excluded from subsequent analyses. The first hierarchical regression involves general external control (independent variable), negative problem orientation (potential mediator) and global social adjustment (dependent variable). The preceding results (Table 5) show that general external control is correlated to negative problem orientation with $(\mathrm{r}[92]=-0.508, p<0.001)$. The regression analyses show that negative problem orientation predicts global social adjustment ( $\beta=-0.442, p<0.001)$ and explains $19.5 \%$ of its variance. The first model shows that general external control significantly predicts global social adjustment $(\beta=-0.337, p<0.001)$ and explains $11 \%$ of its variance. In the second model, negative problem orientation was included in the regression equation before general external control. Following this, general external control no longer predicts global social adjustment $(\beta=-0.151, p>0.05, n s)$. The percentage of explained variance was reduced to $1.7 \%$. This shows a complete mediation effect: the inclusion of negative problem orientation in the regression equation cancels the direct effect of general external control on global social adjustment. A $z$-test was performed and confirmed that effect $(z=3.279, p<0.01)$ (Figure 1). Illustrates this first mediation model.

For the OCD group, the same analyses were conducted with the SPS total score as a potential mediator. The analyses confirmed that the SPS score acts as a mediator in the relationship between PC and adjustment. However, when negative problem orientation is included in the model, this effect is no longer present. Positive problem orientation was not selected as a potential mediator in the OCD group since its correlation coefficient with global social adjustment has a small effect size $(\mathrm{r}=-0.203, \mathrm{p}<0.05)$. Indeed, the exploratory analyses conducted with this group show that this variable does not act as a mediator. The same analyses were conducted for the control group. The same scenario is supported for general external control, negative problem orientation

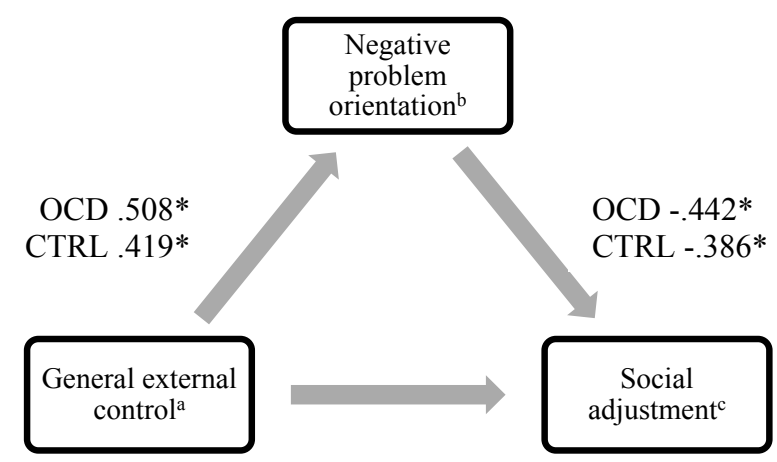

OCD $-.151(-.337 *)$

CTRL $-.099(-.247 *)$

Note: OCD: Obsessive-Compulsive Disorder; CTRL: Control; aHigher scores represent more internal control on an external/internal continuum; bLower scores represent more important difficulties in this dimension; cLower scores represent better social adjustment

${ }^{*} p<0.001$

Figure 1: Mediation effect of negative problem-solving on the relationship between general external control and global social adjustment.

(mediator) and social adjustment. The preceding results (Table 5) show that general external control is correlated to negative problem orientation with $(\mathrm{r}[214]=0.419, \mathrm{p}<0.001)$. The regression analyses show that negative problem orientation predicts global social adjustment ( $\beta=-0.386, p<0.001)$ and explains $14.9 \%$ of its variance. The first model shows that general external control significantly predicts global social adjustment $(\beta=-0.247, p<0.001)$ and explains $6 \%$ of its variance. In the second model, negative problem orientation was included in the regression equation before general external control. Following this, general external control no longer predicts global social adjustment ( $\beta=-0.099, p>0.05, n s)$. The percentage of explained variance was reduced to $0.8 \%$. This shows a complete mediation effect: the inclusion of negative problem orientation in the regression equation cancels the direct effect of general external control on global social adjustment. A $\mathrm{z}$-test was performed and confirmed that effect $(\mathrm{z}=4.266, \mathrm{p}<0.001)$. Figure 1 illustrates this mediation model. For the control group, the same analyses were conducted with the SPS total score as a potential mediator. The analyses confirmed that the SPS score acts as a mediator in the relationship between general external control and global social adjustment. However, as with the OCD group, when negative problem orientation is included in the model, this effect is no longer present. Positive problem orientation was not identified as a mediator for the control group.

\section{Discussion}

The primary goal of this study was to compare personal control (PC) and social problem-solving (SPS) in people with OCD and a comparison group. Regarding personal control (PC), the results have demonstrated that people with OCD show more external control than the controls. These results provide additional support for the conclusions of other studies on the subject [25-27]. It is interesting to note that individuals with OCD present less exaggerated internal control than does the comparison group. Exaggerated internal control was defined earlier as a measure of extreme and unrealistic beliefs in one's PC and represents a dimension of control that is characterized by, at its upper limit, excessive optimism or extreme confidence in one's own power to control events [14]. If the results of the previous studies were found to indicate that the OCD group had more external than internal control, 
we would have expected to observe deleterious patterns for internal control, namely, when we frequently observe an exaggerated perception of responsibility in this group (e.g. belief that one possesses the unique power to provoke or prevent negative material or moral consequences [37]. This pattern was not observed. An examination of the SPS dimensions shows that, as expected, the OCD group has significantly lower scores for global SPS and on all rational problem-solving subscales when compared to the controls. The participants in the OCD group also present more negative problem orientation and less positive problem orientation compared to the comparison group. However, the groups do not differ according to the impulsive/careless and avoidant styles they employ in SPS. Nevertheless, here, non-significant results could be due to the lack of statistical power, making it difficult to make inferences. This would appear to be probable because it is supported by Abolghasemi and Narimani [46], who observed differences in both of these scales for individuals diagnosed with OCD and controls, and if we consider the tendency to avoid, which is frequently observed in this group [58]. Globally, these results are consistent with those of other studies that have observed a general negative pattern of response in different clinical groups when confronted with a problem, especially in anxious people $[59,60]$. However, this study distinguishes itself by a more precise examination of SPS styles and orientations in people with OCD. To our knowledge, only one previous publication has studied this variable in individuals with OCD. Our results support previous findings that people with this disorder present more negative and less positive patterns of SPS compared to controls [46]. The results provide support that important challenges in all dimensions of the SPS are observed in people with OCD, who are thus particularly vulnerable and ineffective when faced with everyday life difficulties. The second goal of this study was to examine the relationship between PC and global social adjustment. The results showed that for both groups, the more the internal control increases, the more the adjustment increases. These results are also consistent with other studies arguing that more external control is associated with several psychopathologies [18,61-63] and that more internal control is beneficial to psychological adjustment [20,21]. Previous studies have argued that a weak sense of control is related to more severe obsessive-compulsive symptoms [28-30]. The current study specifies that an external locus of control also negatively affects an individuals' overall social functioning.

This research also aimed to analyze the relationship between SPS and social adjustment by comparing it across individuals in the OCD and control groups. The results show that for both groups, better global SPS is associated with better social adjustment. This observation is also consistent with the scientific literature suggesting that problem-solving deficits are associated with several variables related to psychological distress or adjustment difficulties [32,36]. This study's contribution lies in its support of the precise role of SPS on social adjustment in people with OCD.

The results of this study are also distinguished by examining the relationships between the different dimensions of SPS and social adjustment. For both groups, an increase in negative problem orientation is associated with a decrease in social adjustment. Positive problem orientation is also associated with adjustment in both groups; the higher the positive problem orientation is, the better the social adjustment is. Different studies found that problem orientations, especially negative orientation, are robust predictors of multiple adjustment and well-being variables [37-39,45]. The results of this study support that this relationship is also observable in people with OCD. In addition, negative problem orientation, being predominant in this group, greatly affects their adjustment capacity.
The impulsive/careless style is also associated with adjustment in both groups with a tendency towards impulsivity, which is associated with poorer adaptation. The avoidance style is not significantly associated with adjustment for the OCD group but is associated with adjustment for the controls. The tendency toward avoidance is associated with poorer adaptation for this group. For the control group, the scales for problem definition and formulation as well as solution implementation and verification are also related to global adjustment; an improvement in these scales is associated with improvement in social adjustment for people with OCD. These results are similar to those of Chang et al. [45], who also found that other dimensions of SPS significantly but minimally contribute to well-being compared to negative problem orientation. Nevertheless, it was expected that the concrete capabilities of solving problems would have had a greater impact on adjustment. However, the present results only partially support this hypothesis; specific SPS abilities affect adjustment only for the comparison group. While these abilities may be less developed in the OCD group compared to the control group, they do not affect adjustment. It is possible that since negative problem orientation is predominant in the OCD group, the negative impact of these specific abilities is diluted. Indeed, although it modulates problem-solving abilities, problem orientation operates in the first step of problemsolving and influences both the emotional response to problems and the motivation/confidence to solve them [31]. Accordingly, negative problem orientation appears to have a greater impact on adjustment than more specific problem solving skills. This research also aimed to verify the links between the PC and SPS dimensions. The results show that for both groups, an increase in internal control increases global SPS skills. They also demonstrate that an increase of internal control is associated with a reduction of negative orientation and an increase in positive orientation for both groups.

\section{Conclusion}

A general conclusion emerges from these results: increasing internal control is generally associated with more positive and constructive SPS patterns. These results are consistent with the scientific literature describing a relationship between internal control and an increase in problem-solving activities in the general population $[33,48,49]$. It was also previously suggested that more external control may be observed in people with OCD, which would potentially undermine their problem-solving skills and consequently have a deleterious effect on their social adjustment. Associations were also measured between the PC and SPS subscales. For both groups, more internal control is associated with reduced avoidance and impulsivity towards problems. The results also show that for both groups, more internal control is associated with improved rational problem-solving. Internal control appears to have a more important positive impact on the problem definition and formulation as well as on the solution implementation and verification subscales. These results suggest that PC not only affects the emotional reaction but also the problem-solving strategies used. Ultimately, this study has examined the mediational role of SPS in the relationship between PC and global adjustment; the mediation hypotheses that were made in this regard are supported. Indeed, the results show that for both groups, negative problem orientation acts as a mediator in the relationship between PC and social adjustment. SPS is also a mediator in the same relationship but its effect disappears when negative problem orientation is included in the model. The global SPS score was already identified in different groups as a mediator for the relationships between different variables that may have an impact on adjustment (e.g. adverse life events, personal problems, stress, perfectionism) and different adjustment or psychological well-being 
variables $[39,40,45]$. Our results, however, specify that this link is also true for the relationship between PC and adjustment for both people with $\mathrm{OCD}$ and the controls. The results also suggest that a negative problem orientation towards social problems, by acting as a mediator, more strongly affects this relationship. The importance of this variable in understanding the psychological adjustment of several groups has already been established [37-39,44]. Our results thus confirm that negative orientation is central in determining social adjustment in people with OCD.

The results of this study highlight several conclusions. First, PC was identified as affecting SPS and adjustment. SPS also directly affects social adjustment. In addition, the impact of control on adjustment is also indirect because it is involved in SPS, particularly negative problem orientation. This orientation is a cognitive and emotional dysfunctional pattern that involves various general trends, including the disposition to observe a problem as a significant threat to well- being, doubt one's abilities to solve problems successfully and become frustrated when confronted with a problem [31]. Negative problem orientation is a measure of motivation and confidence towards SPS skills. In 1977, Bandura stressed the problems of only considering the PC, suggesting that it is not sufficient to believe that one's actions are responsible for the results but that it is also essential to consider a person's perceptions of his or her ability to control his or her actions. Our results confirm this idea that confidence in one's problem- solving skills is central. Our results support a strong association between SPS and social adjustment in people with OCD. It would therefore be interesting to include SPS training in the treatment for OCD. The integration of therapeutic interventions on SPS skills should first address negative problem orientation. This type of intervention would be congruent with what is done in the treatment of other disorders, such as schizophrenia, to improve social functioning [64]. This study also has limitations. First, its cross-sectional nature does not allow conclusions to be drawn as to the direction of the studied relationships. Thus, longitudinal research would be interesting to conduct in future studies. Second, a limitation of the SAS-SR must be raised. Indeed, the codification of the scales of the questionnaire does not consider the negative weight of the participants who have not answered to the questions concerning occupation, such as those who are unemployed or not in school and those who do not see themselves as homemakers. Therefore, people who do not have an occupation because of excessive dysfunction had no impact on our results, which could create a bias. A second set of limitations concerns sampling and its evaluation.

\section{Limitations}

The firstlimitation is related to the absence of some sociodemographic data for the control group, which prevents any comparison of these variables with the OCD group. Additionally, no psychological evaluation has been conducted for the participants in the control group because of pragmatic limitations of feasibility. It is possible that this sample, if it presents a high prevalence of psychological problems, may bias the results. Similarly, knowing that the presence of primary and comorbid depression is associated with significant adjustment problems and can sometimes explain greater variance in difficulties other than the main psychological problem [65] measuring depression would have been an interesting addition and should be considered for future research. Although this study does contain methodological limitations, it allows an innovative and interesting view of several variables that have an impact on adjustment in people with OCD. It primarily examined the links between SPS and social adjustment. SPS has proven to be an important variable in understanding the adjustment difficulties that are observable in people with OCD. Considering the dimensions of
PC and SPS during evaluation and treatment should allow clinicians to positively and directly influence adjustment abilities and increase the patients' rehabilitation opportunities in various areas of their social functioning. It would therefore be important to expand this research by considering the impact of SPS dimensions on the relationship between symptoms and adjustment.

\section{References}

1. American Psychiatric Association (2013) Diagnostic and statistical manual of mental disorders: DSM-5, Washington.

2. Rosa AC, Diniz JB, Fossaluza V, Torres AR, Fontenelle LF, et al. (2012) Clinical correlates of social adjustment in patients with obsessive-compulsive disorder. J Psychiatr Res 46: 1286-1292.

3. Karno M, Golding JM, Sorenson SB, Burnam MA (1988) The epidemiology of obsessive-compulsive disorder in five US communities. Arch Gen Psychiatry 45: 1094-1099.

4. Leon AC, Portera L, Weissman MM (1995) The social costs of anxiety disorders Br J Psychiatry 166: 19-22.

5. Regier DA, Farmer ME, Rae DS, Myers JK, Kramer M, et al. (1993) One-month prevalence of mental disorders in the United States and sociodemographic characteristics: The Epidemiologic Catchment Area program. Acta Psychiatrica Scandinavica 88: 35-47.

6. Stein DJ, Bruun RD, Josephson SC, Hollander E (1991) Obsessional severity in Tourette's syndrome. J Clin Psychiatry 52: 388.

7. Bystritsky A, Liberman RP, Hwang S, Wallace CJ, Vapnik T, et al. (2001) Socia functioning and quality of life comparisons between obsessive-compulsive and schizophrenic disorders. Depress Anxiety 14: 214-218.

8. Calvocoressi L, Libman D, Vegso SJ, McDougle CJ, Price LH (1998) Global functioning of inpatients with obsessive-compulsive disorder, schizophrenia and major depression. Psychiatr Serv 49: 379-381.

9. Didie ER, Pinto A, Mancebo M, Rasmussen SA, Phillips KA et al. (2007) A comparison of quality of life and psychosocial functioning in obsessivecompulsive disorder and body dysmorphic disorder. Ann Clin Psychiatry 19 181-186.

10. Grenier S, Préville M, Boyer R, O'Connor K (2009) Prevalence and correlates of obsessive compulsive disorder among older adults living in the community. $\mathrm{J}$ Anxiety Disord 23: 858-865.

11. Lochner C, Mogotsi M, du Toit PL, Kaminer D, Niehaus D J, et al. (2003) Quality of life in anxiety disorders: A comparison of obsessive-compulsive disorder social anxiety disorder and panic disorder. Psychopathology 36: 255-262.

12. Weissman MM (1999) Social Adjustment Scale-self Report (SAS-SR): User's Manual. Multi-Health Systems Inc., North Tonawanda, NY.

13. Rotter JB (1966) Generalized expectancies for internal versus external contro of reinforcement. Psychological Monographs: General and Applied 80: 1-28.

14. Berrenberg JL (1987) The belief in personal control scale: A measure of Godmediated and exaggerated control. J Pers Assess 51: 194- 206.

15. Bandura A (1977) Self-efficacy: Toward a unifying theory of behavioral change. Psychol Rev 84: 191-215.

16. Skinner EA (1996) A guide to constructs of control. J Pers Soc Psychol 71: 549-570.

17. Njus DM, Brockway JH (1999) Perceptions of competence and locus of control for positive and negative outcomes: Predicting depression and adjustment to college. Pers Individ Dif 26: 531-548.

18. Holder EE, Levi DJ (1988) Mental health and locus of control: SCL-90-R and Levenson's IPC scales. J Clin Psychol 44: 753-755.

19. Shapiro Jr DH, Schwartz CE, Astin JA (1996) Controlling ourselves, controlling our world: Psychology's role in understanding positive and negative consequences of seeking and gaining control. Am Psychol 51: 1213-1230.

20. Leung C (2001) The psychological adaptation of overseas and migrant students in Australia. Int J Psychol 36: 251-259.

21. Caldwell RA, Pearson JL, Chin RJ (1987) Stress-moderating effects: Socia support in the context of gender and locus of control. Pers Soc Psychol Bull 13: $5-17$. 
Citation: Aubut M, Belanger C (2017) Personal Control, Social Problem-Solving and Social Adjustment in Obsessive-Compulsive Disorder. J Psychol Abnorm 6: 156. doi: 10.4172/2471-9900.1000156

22. Wortman CB (1976) Causal attribution and personal control. New directions in attribution research, Erlbaum, Hinsdale, NJ.

23. Estrada L, Dupoux E, Wolman C (2006) The relationship between locus of control and personal-emotional adjustment and social adjustment to college life in students with and without learning disabilities. Coll Stud J 40: 43-54.

24. Clark DA (2004) Cognitive-behavioral therapy for OCD. New York, NY, US

25. Gillan CM, Morein-Zamir S, Durieux AMS, Fineberg NA, Sahakian BJ, et al. (2014) Obsessive-compulsive disorder patients have a reduced sense of control on the illusion of control task. Front Psychol 5: 204.

26. Moulding R, Doron G, Kyrios M, Nedeljkovic M (2008) Desire for control sense of control and obsessive-compulsive checking: An extension to clinical samples. J Anxiety Disord 22: 1472-1479.

27. Kennedy BL, Lynch GV, Schwab JJ (1998) Assessment of locus of control in patients with anxiety and depressive disorders. J Clin Psychol 54: 509-515.

28. McLaren S, Crowe SF (2003) The contribution of perceived control of stressful life events and thought suppression to the symptoms of obsessive-compulsive disorder in both non-clinical and clinical samples. J Anxiety Disord 17: 389-403.

29. Moulding R, Kyrios M (2007) Desire for control, sense of control and obsessivecompulsive symptoms. J Anxiety Disord 31: 759-772.

30. Altin M, Karanci AN (2008) How does locus of control and inflated sense of responsibility relate to obsessive-compulsive symptoms in Turkish adolescents? J Anxiety Disord 22: 1303-1315.

31. D'Zurilla TJ, Nezu AM, Maydeu Olivares A (2002) Social problem-solving inventory-revised (SPSI-R). Multi-Health Systems Inc., North Tonawanda, NY.

32. Heppner PP, Witty TE, Dixon WA (2004) Problem-solving appraisal and human adjustment: A review of 20 Years of research using the problem-solving inventory. Counsel Psychol 32: 344-428.

33. Nezu AM (1985) Differences in psychological distress between effective and ineffective problem solvers. J Counsel Psychol 32: 135-138.

34. Chang EC, D'Zurilla TJ (1996) Relations between problem orientation and optimism, pessimism and trait affectivity: A construct validation study. Behav Res Ther 34: 185-194.

35. Elliott TR, Herrick SM, Macnair RR, Harkins SW (1994) Personality correlates of self-appraised problem-solving ability: Problem orientation and trait affectivity. $J$ Pers Assess 63: 489-505.

36. Nezu AM (2004) Problem-solving and behavior therapy revisited. Behav Ther 35: $1-33$

37. Dugas MJ, Letarte H, Rhéaume J, Freeston MH, Ladouceur R (1995) Worry and problem-solving: Evidence of a specific relationship. Cogn Ther Res 19: 109-120.

38. Gosselin P, Dugas MJ, Ladouceur R (2002) Worry and problem-solving: The role of negative problem orientation. Journal de Thérapie Comportementale et Cognitive 12: 49-58.

39. Kant GL, D'Zurilla TJ, Maydeu-Olivares A (1997) Social problem-solving as a mediator of stress-related depression and anxiety in middle-aged and elderly community residents. Cogn Ther Res 21: 73-96.

40. Chang EC (2002) Examining the link between perfectionism and psychological maladjustment: Social problem-solving as a buffer. Cogn Ther Res 26: 581595.

41. Nezu AM, Ronan GF (1988) Social problem-solving as a moderator of stressrelated depressive symptoms: A prospective analysis. J Counsel Psychol 35 134-138.

42. Nezu AM (1986) Negative life stress and anxiety: Problem-solving as a moderator variable. Psychol Rep 58: 279-283.

43. Nezu AM, Nezu CM, Saraydarian L, Kalmar K, Ronan GF (1986) Socia problem-solving as a moderating variable between negative life stress and depressive symptoms. Cogn Ther Res 10: 489-498.
44. Bell AC, D'Zurilla TJ (2009) The influence of social problem-solving ability on the relationship between daily stress and adjustment. Cogn Ther Res 33: 439 448

45. Chang EC, D'Zurilla TJ, Sanna LJ (2009) Social problem-solving as a mediato of the link between stress and psychological well-being in middle-adulthood. Cogn Ther Res 33: 33-49.

46. Abolghasemi A, Narimani M (2008) A comparison of social problem solving in patients with OCD, PTSD, PD and non-patient controls: To control of the intolerance of uncertainty. Res J Biol Sci 3: 683-689.

47. Fergus TA, Wu KD (2010) Do symptoms of generalized anxiety and obsessivecompulsive disorder share cognitive processes? Cogn Ther Res 34: 168.

48. Dijkstra MTM, Beersma B, Evers A (2011) Reducing conflict-related employee strain: The benefits of an internal locus of control and a problem-solving conflict management strategy. Work Stress 25: 167-184.

49. Collins K, Luszcz M, Lawson M, Keeves J (1997) Everyday problem-solving in elderly women: Contributions of residence, perceived control and age. Gerontologist 37: 293-302.

50. Reese FL, Kliewer W, Suarez T (1997) Control appraisals as moderators of the relationship between intrusive thoughts and coping. J Appl Soc Psychol 27: $1131-1145$

51. Baumgardner AH, Heppner PP, Arkin RM (1986) Role of causal attribution in personal problem-solving. J Pers Soc Psychol 50: 636-643.

52. First MB, Spitzer RL, Gibbon M, Williams JB (1997) Structured clinical interview for DSM-IV Axis I Disorders - Patient Edition (SCID-I/P, version 2.0, 4/97 revision) 722 West 168th Street, New York, NY 10032.

53. Waintraub L, Guelfi JD, Lancrenon S, Rouillon F (1995) Validation du questionnaire d'adaptation sociale de M. Weissman dans sa version française. Ann Med Psychol 153: 274-278.

54. Weissman MM, Bothwell S (1976) Assessment of social adjustment by patient self-report. Arch Gen Psychiatry 33: 1111-1115.

55. Weissman MM (1999) Social adjustment scale-self report (SAS-SR): User's manual. Multi-Health Systems Inc., North Tonawanda, NY.

56. Erdfelder E, Faul F, Buchner A (1996) GPOWER: A general power analysis program. Behavior Research Methods, Instruments and Computers 28: 1-11.

57. Baron RM, Kenny DA (1986) The moderator-mediator variable distinction in social psychological research: Conceptual, strategic and statistical considerations. J Pers Soc Psycho 51: 1173-1182.

58. Starcevic V, Berle D, Brakoulias V, Sammut P, Moses K, et al. (2011) The nature and correlates of avoidance in obsessive-compulsive disorder. Aust $\mathrm{N} \mathrm{Z}$ J Psychiatry 45: 871-879.

59. Ladouceur R, Blais F, Freeston MH, Dugas MJ (1998) Problem-solving and problem orientation in generalized anxiety disorder. J Anxiety Disord 12: 139152

60. Nezu AM, Carnevale GJ (1987) Interpersonal problem-solving and coping reactions of Vietnam veterans with posttraumatic stress disorder. J Abnorm Psychol 96: 155-157.

61. Burger JM (1984) Desire for control, locus of control and proneness to depression. J Pers 52: 71-89.

62. Ganellen RJ, Blaney PH (1984) Stress, externality and depression. J Pers 52 326-337.

63. Layton C (1985) The relationship between externality and general nonpsychotic psychiatric morbidity in normal males. Percept Mot Skills 61: 746.

64. Liberman RP, Eckman TA, Marder SR (2001) Training in social problem-solving among persons with schizophrenia. Psychiatr Serv 52: 31-33.

65. Masellis M, Rector NA, Richter MA (2003) Quality of life in OCD: Differentia impact of obsessions, compulsions and depression comorbidity. Can J Psychiatry 48: 72-77 